\title{
Exogenous orexin-A downregulates luteinizing hormone secretory activity in prepubertal female rats
}

\author{
Lidia Martyńska' , Alina Gajewska ${ }^{2}$, Magdalena Chmielowska' ${ }^{1}$, Małgorzata Kalisz ${ }^{1}$, Anna Litwiniuk ${ }^{1}$, \\ Wojciech Bik ${ }^{1}$, Bogusława Baranowska ${ }^{3}$ \\ ${ }^{1}$ Department of Neuroendocrinology, Centre of Postgraduate Medical Education, Warsaw, Poland \\ ${ }^{2}$ Department of Animal Physiology, The Kielanowski Institute of Animal Physiology and Nutrition, Polish Academy of Sciences, \\ Jabtonna, Poland \\ ${ }^{3}$ Department of Neurology, Faculty of Medical Sciences, Medical University of Warsaw, Warsaw, Poland
}

\begin{abstract}
Introduction: Orexin-A is a neuropeptide synthesized in the lateral hypothalamus. Orexin-A immunoreactive fibres overlap distribution with GnRH neurons. In adult rats, orexin A is known to affect LH secretion via GnRH release modulation. Because data concerning the impact of orexin-A on the hypothalamo-pituitary axis activity are limited, we focused on the involvement of orexin-A and receptors of NPY in the modulation of LH release and LH subunit $\beta(L h b)$ mRNA expression in prepubertal female rats.

Material and methods: Forty immature female Wistar rats were divided into 4 groups and received 2 intracerebroventricular (icv) microinjections of: 1 - artificial cerebrospinal fluid (CSF) (controls); 2 - CSF followed by orexin A; 3 - selective NPY receptor antagonist (BIBP) followed by CSF; 4 - BIBP followed by orexin A. One hour after the last microinjection, all rats were decapitated. Trunk blood was collected, and serum was stored at $-20^{\circ} \mathrm{C}$ for the LH RIA examination. The adenohypophysis was immediately excised, flash-frozen, and kept at $-80^{\circ} \mathrm{C}$ for RNA extraction. Real-time PCR amplification was carried out, and relative Lhb gene expression was calculated.

Results: In comparison to the CSF-treated controls with a mean LH serum concentration of $0.40 \pm 0.02 \mathrm{ng} / \mathrm{mL}$, the mean LH serum level was diminished both after orexin-A $(0.27 \pm 0.01 \mathrm{ng} / \mathrm{mL})$ and after BIBP $(0.30 \pm 0.02 \mathrm{ng} / \mathrm{mL})$ icv microinjections. In the presence of BIBP, orexin-A more effectively inhibited LH release $(0.20 \pm 0.01 \mathrm{ng} / \mathrm{mL})$ when compared to the BIBP-treated group.

Orexin-A and BIBP exerted a consistent inhibitory effect on $L h b$ mRNA expression levels in the anterior pituitary gland. In comparison to the CSF-treated controls, orexin-A, and BIBP-treated females responded with, respectively, $35 \%$ and $40 \%$ reduction of $L h b$ mRNA expression. Orexin-A and BIBP co-administration evoked a further reduction of Lhb gene transcriptional activity.

Conclusions: Orexin-A exerts a down-regulatory effect on LH synthesis and release in immature female rats. Considering that Y1R-oriented down-regulation of endogenous NPY activity did not reverse the suppressive effect of exogenous orexin-A, it might be suggested that NPY and orexin A systems can operate independently to affect gonadotropin activity in the anterior pituitary of the immature female rats. (Endokrynol Pol 2021; 72 (3): 238-242)
\end{abstract}

Key words: orexin A; LH; Lhb mRNA; NPY Y1 receptor antagonist; prepubertal female rats

\section{Introduction}

Due to its principal role in the regulation of reproductive functions, the hypothalamic-pituitary-gonadal (HPG) axis is under the influence of several hormonal and neuronal cross-talks, including hypothalamic neuropeptide orexin-A.

Orexin-A is a 33-amino acid neuropeptide whose primary structure is completely conserved in humans, rats, mice, and other mammals. Orexin-A is produced by proteolytic cleavage of the 130-131 amino acids prepro-orexin, a highly conserved polypeptide with $75 \%$ amino acid sequence identity $[1,2]$. In the brain, orexin-A is synthesized by specific neurons localized in the lateral hypothalamus and perifornical area. These neurons contain a variety of receptors enabling them to respond to an array of signals related to environmen- tal, physiological, and emotional stimuli. They project broadly to the entire central nervous system (CNS) [3]. As a multitasking peptide, orexin-A is involved in a spectrum of physiological functions including regulation of feeding behaviour, autonomic/neuroendocrine functions, and sleep/wakefulness states in mammals [4].

It is well established that the hypothalamic neuropeptide $\mathrm{Y}$ (NPY) neural network is involved in mediating orexin-A-induced feeding behaviour [5]. Indeed, orexin-A neurons were shown to form direct synaptic contacts with NPY neurons in the paraventricular nucleus and with NPY/Agouti-related peptide neurons within the arcuate nucleus [6], which in turn enables the NPY system to mediate orexin-A-induced food intake. Furthermore, central orexin-A infusion was shown to induce NPY expression in the arcuate nucleus [7] whereas the orexin-A orexigenic effect was reversed 
in the presence of centrally applied selective antagonist of NPY Y1 receptor [8, 9]. Noticeably, disturbances in orexin-A activity were found in patients with severe metabolic disorders [10].

It is well recognized that the gonadotropin-releasing hormone $(\mathrm{GnRH})$ neuronal system within the septo-preoptic and the arcuate nucleus-median eminence region shares an overlapping distribution with orexinA immunoreactive fibres, which indicates that orexinA could be involved in the modulation of pituitary luteinizing hormone secretion via regulation of $\mathrm{GnRH}$ release [11-13]. A modulatory impact of orexin-A on pituitary gonadotropin activity could be also mediated by the NPY neuronal system. Data from the literature indicate that administration of orexin-A significantly reduced the mean $\mathrm{LH}$ concentration and $\mathrm{LH}$ pulse frequency in female rats, whereas co-administration of NPY antibody with orexin-A significantly restored the suppressive effect of orexin-A on the mean LH concentration and LH pulse frequency [14].

Because results concerning the impact of orexinA on the HPG axis are predominantly derived from research on adult female rodents, there is scarce evidence of its role in prepubertal female rats. Considering the prepubertal stage of development in the context of specific GnRH neuronal network sensitivity for central and peripheral hormonal signals [15], a distinct orexinA sensitivity in the hypothalamo-pituitary axis cannot be excluded in these animals.

Therefore, in this study, we directly examined the $\mathrm{LH}$ release and $\mathrm{Lhb}$ gene transcriptional response to orexin application and evaluated how this response changes in the presence of a specific NPY receptor antagonist in immature female Wistar rats.

\section{Material and methods}

\section{Chemicals}

Orexin-A and BIBP 3236 - NPY Y1 receptor antagonist were obtained from Sigma-Aldrich (Saint Louis, MO, USA), ketamine (Bioketan Vetoquinol 10\%), and xylazine (Sedazin 2\%) from Biowet, Poland, and ketoprofen (Ketonal, $50 \mathrm{mg} / \mathrm{mL}$ ) from Lek pharmaceuticals d.d, Slovenia. Dental cement (Duracyl Plus) was from Spofa Dental, Czech Republic.

\section{Animals and surgical protocol}

Forty immature 25-day-old female Wistar rats (weighting 90-110 g) were obtained from Laboratory Animal Breeding (Warsaw, Poland). To adapt to the experimental conditions, the animals were kept for 7 days under controlled conditions of light (14:10 h light/dark cycle, lights on $06: 00 \mathrm{~h})$, temperature $\left(23 \pm 1^{\circ} \mathrm{C}\right)$, and humidity $(45-55 \%)$, with free access to tap water and standard pelleted food (Murigran, Poland). After this adaptation period, all rats were anaesthetized (ketamine + xylazine $(5 \mathrm{mg}+2 \mathrm{mg} / 100 \mathrm{~g}$ body weight (b.w.), respectively). Then, a permanent stainless-steel gauge was implanted stereotaxically into the third ventricle. Coordinates were selected according to the rat brain atlas [16]. The gauge was cemented with Duracryl in place, and its location was confirmed by observing the flow of cerebrospinal fluid. After surgery, all animals received analgetic (Ketonal $0.5 \mathrm{mg} / 100 \mathrm{~g}$ b.w.) for 3 consecutive days. They were left to recover for 7 days before the start of the experiment.

\section{Experimental design}

Rats were randomly assigned to experimental groups $(n=10)$. The experiments started on day 8 when the animals were connected to an automatic CMA/100 pump (CMA Microdialysis AB, Stockholm, Sweden) with $45 \mathrm{~cm}$ long silicon tubing (ID, $0.5 \mathrm{~mm}$, OD, $1 \mathrm{~mm}$ ), allowing them to move freely. Orexin-A and BIBP-3226 (BIBP) were dissolved in artificial cerebrospinal fluid (CSF) to obtain a concentration of $1 \mu \mathrm{g} / 5 \mu \mathrm{L}$. All animals were given 2 intracerebroventricular (icv) microinjections applied with a 30-min. interval. Depending on the group, rats received the following:

1 - CSF (controls);

2 - CSF followed by orexin-A;

3 - BIBP followed by CSF

4 - BIBP followed by orexin-A.

\section{Sample collection}

One hour after the last microinjection, all rats were killed by decapitation under ketamine + xylazine anaesthesia. The anterior pituitary was immediately excised, flash-frozen in liquid nitrogen, and finally kept at $-80^{\circ} \mathrm{C}$ for subsequent RNA extraction. Trunk blood was centrifuged in dry tubes, and the serum was stored at $-20^{\circ} \mathrm{C}$ for $\mathrm{LH}$ assessment by RIA.

All experimental procedures were conducted following the Polish Guide for the Care and Use of Animals and were approved by the 4th Local Animal Use and Care Ethics Committee, National Medicines Institute, Warsaw, Poland.

Quantitative reverse transcriptase PCR (RT-qPCR) Total RNA was extracted using the TRIZOL reagent (Invitrogen, USA) following the manufacturer's protocol. cDNA was synthesized from $800 \mathrm{ng}$ of the total RNA using anchored random hexamer primers and Moloney murine leukaemia virus reverse transcriptase according to the protocol of DyNAmo TM cDNA synthesis kit (Finnzymes, Finland). Real-time PCR amplification was carried out using SYBRGreen 2-step qRT-PCR kit (Finnzymes, Finland) according to the manufacturer's protocol. Relative $L h b$ gene expression was calculated using the comparative quantitation option of Rotorgene-Q software (Qiagen, USA). To compensate for the variation in cDNA concentrations and the PCR efficiency between tubes and endogenous control, the rat glyceraldehyde3-phosphate dehydrogenase ( $\mathrm{rGapdh}$ ) gene was quantified in each sample and used for normalization. The average relative $L h b$ gene expression of the control group was set to 1.0. Pairs of primers specific for the $\mathrm{rLhb}$ gene and primers specific for the reference rGapdh gene were designed to span over intron sequences using the Primer3 open-source software [17] (IDT PrimerQuest; Integrated DNA Technologies Inc., USA). Primer specificity was confirmed by a BLAST software-assisted search of a nonredundant nucleotide sequence database (National Library of Medicine, USA). Specific primer sequences were as follows:

rLhb: Acc No: NM 001033975.1: F (154): CCTGGCTGCAGAGAATGAGT

R (286): GTAGGTGCACACTGGCTGAG, amplicon size: 133 bp rGapdh: Acc No: NM_017008.4: F (901): GAGGACCAGGTTGTCTCCTG

R (1061): ATGTAGGCCATGAGGTCCAC; amplicon size 161 bp

\section{Radioimmunoassay}

Rat serum LH was measured by RIA using antibodies and LH preparation supplied by Dr. A.F. Parlow and NIDDK (USA). Values were expressed in terms of rat LH-RP-3 reference preparation. Intra-assay variation for $\mathrm{LH}$ was less than $7 \%$. 


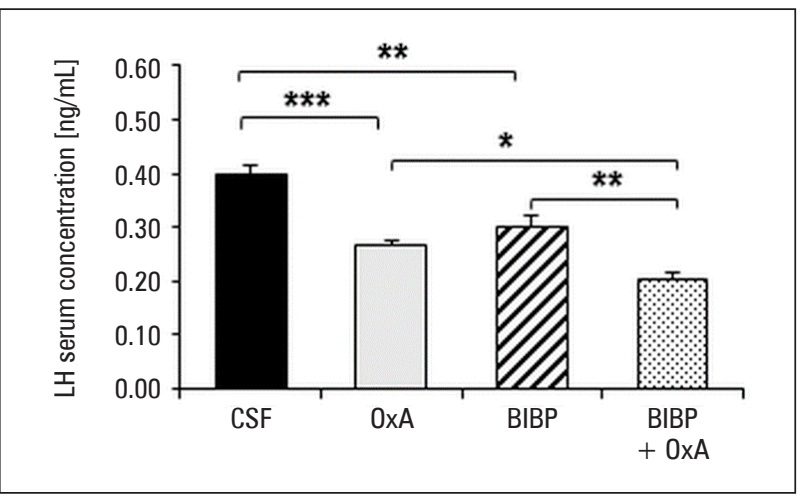

Figure 1. Effects of intracerebroventricular (icv) infusion of orexin-A (OxA) and selective NPY receptor antagonist (BIBP) on mean luteinizing hormone $(L H)$ serum levels in prepubertal female rats. All values are mean \pm SEM for 10 rats per group. ${ }^{*} p<0.05,{ }^{* *} p<0.01,{ }^{* *} p<0.001$.

\section{Statistical analysis}

All results were analysed with Statistica 6.0 PL (StatSoft Inc., USA). Differences resulting in $\mathrm{p}<0.05$ were considered statistically significant.

RIA results were expressed as the means \pm SEM. One-way analysis of variance (ANOVA) followed by Tukey's post-hoc multiple comparison test was performed.

All results concerning comparisons of specific $L h b$ mRNA levels were expressed as a ratio to a calibrator that was chosen to be the CSF microinjected group and presented as the mean values \pm SEM. Statistical evaluations were carried out using nonparametric Kruskal-Wallis rank test, followed by Mann-Whitney U test.

\section{Results}

\section{Changes of serum LH concentration: an effect} of icv orexin- $A$ and BIBP microinjections

In comparison to the CSF-treated controls with mean LH serum concentration of $0.40 \pm 0.02 \mathrm{ng} / \mathrm{mL}$, mean LH serum concentration was diminished both after separate orexin-A $(0.27 \pm 0.01 \mathrm{ng} / \mathrm{mL})$ or BIBP $(0.30 \pm 0.02$ $\mathrm{ng} / \mathrm{mL}$ ) icv microinjections. In the presence of NPY Y1 receptor antagonist, orexin-A inhibited LH release more effectively $(0.20 \pm 0.01 \mathrm{ng} / \mathrm{mL})$ when compared to the BIBP-treated group (Fig. 1).

\section{Modulatory effect of orexin-A and BIBP on Lhb mRNA expression}

When applied separately, orexin-A and BIBP exerted a consistent inhibitory effect on $L h b$ mRNA expression level. In comparison to the CSF-treated controls, orexinA- and BIBP-treated females responded, respectively, with $35 \%$ and $40 \%(\mathrm{p}<0.05)$ reduction of Lhb mRNA expression in the anterior pituitary gland (Fig. 2). Orexin-A and BIBP co-administration evoked a further reduction of $L h b$ gene transcriptional activity. In detail, in these animals, Lhb gene expression was reduced by $36 \%$ when compared to the orexin-A-treated group $(\mathrm{p}<0.05)$

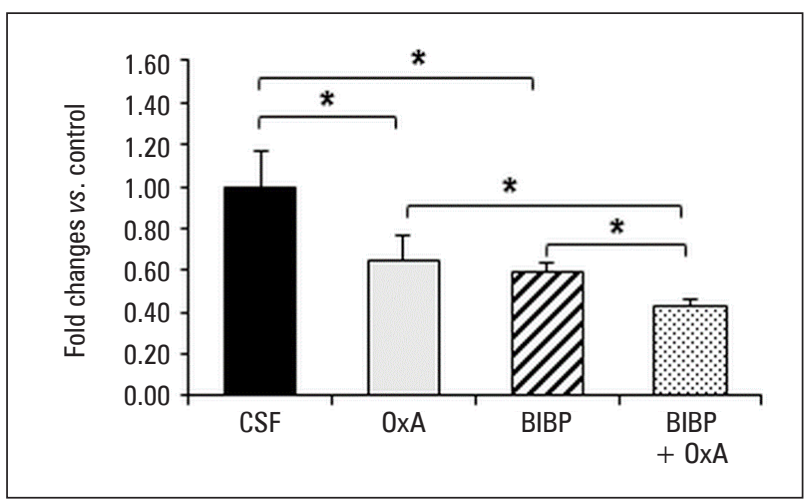

Figure 2. Effects of intracerebroventricular (icv) infusion of orexin- $A$ $(O x A)$ and selective NPY receptor antagonist (BIBP) on relative Lhb $m R N a$ expression in adenohypophysis of prepubertal female rats. Data are expressed as fold changes vs. CSF-treated group, and values represent the mean \pm SEM. CSF - cerebrospinal fluid; * $p<0.05$

and by $29 \%$ in comparison to the BIBP-injected group $(\mathrm{p}<0.05)$ (Fig. 2).

\section{Discussion}

Our data revealed that exogenous orexin-A down-regulated gonadotropic activity in prepubertal female rats. The observed effect concerned both LH release as well as $L h b$ subunit mRNA expression level. These results may indicate that this specific response is a consequence of the impact of orexin-A on endogenous GnRH release.

As reported in the literature, approximately $80 \%$ of GnRH neurons in rats express the orexin-1 receptor (OX1R) and have direct synaptic contact by orexin fibres providing an anatomical basis for orexin-A-induced GnRHneuronal activity regulation [18]. Also, electrophysiological studies on female mice indicate that orexin-A reduces the overall firing rate by suppressing spike initiation and burst maintenance in GnRH neurons [19]. Although data concerning the influence of orexin-A on reproductive axis activity in immature rats are scarce, a decrease of mean plasma concentration and $\mathrm{GnRH} / \mathrm{LH}$ pulse amplitudes in prepubertal female and male rats receiving intracerebroventricular orexin-A infusion has been reported previously [20]. Moreover, the presence of close appositions found between orexin-A immunoreactive varicosities and $\mathrm{GnRH}$ cell bodies in the rostral preoptic area (POA) further support the hypothesis that the orexin-induced effect on LH release may involve direct actions on GnRH neurons [21]. In contrast, our earlier in vitro studies revealed that orexin-A applied to primary anterior pituitary cells isolated from immature female rats increased LH secretion, but when orexin-A was given together with exogenous $\mathrm{GnRH}$ we did not observe this effect [22]. The discrepancy between in vitro and in vivo results suggests that, in pre- 
pubertal females, orexin A might affect the Lhb mRNA level in a specific-site-dependent manner. In adult rats, the gonadal steroid status determines the specificity of orexin-A impact on gonadotropic activity. Orexin-A infused centrally was shown to be involved in the generation of ovarian steroid-induced $\mathrm{LH}$ and prolactin surges in ovariectomized rats [23]. Furthermore, data obtained from steroid-unprimed rats revealed orexin-A down-regulatory input on LH release [24].

In the present research, 35-day-old prepubertal females were subjected to intracerebroventricular infusions. Although their gonadal steroid serum concentration was not determined in our study, it has been reported that female rats between day 25 and puberty exhibit low oestradiol serum levels [25]. Subsequently, a low oestradiol serum concentration, characteristic of this period of development, might significantly contribute to the orexin-A-induced inhibitory effect exerted on gonadotropin activity that was observed in our research.

Moreover, site-specific effects of orexin-A were also reported by Small et al. In detail, orexin-A injected into the rostral preoptic area at the level of organum vasculosum of the lamina terminalis (OVLT) stimulated LH release in oestradiol benzoate-treated ovariectomized rats. When orexin A was given into the medial POA or the arcuate/median eminence, it inhibited the oestradiol/progesterone-induced LH surge in ovariectomized rats and reduced LH levels in females untreated with ovarian steroids [21]. Also, it should be taken into account that NPY was reported to mediate the inhibitory effect of orexin-A-injected intracerebroventricularly on pulsatile LH secretion in adult ovariectomized female rats. Indeed, co-administration of NPY antibody with orexin-A significantly restored the suppressive effect of orexin-A on LH concentration and pulse frequency [14]

If NPY mediates the suppressive effect of hypothalamic orexin on pulsatile GnRH secretion in adult animals, then the Y1R-mediated influence on orexin-A-evoked gonadotropin synthesis and release should be likewise assumed in immature females. Hence, to evaluate $\mathrm{Y} 1 \mathrm{R}$ involvement in orexin regulatory inputs exerted at the pituitary level, a pharmacological approach based on the selective antagonist infusion was applied in our study. Surprisingly, a reduced concentration of LH in serum accompanied by diminished $L h b$ mRNA expression not only showed the effectiveness of BIBP in affecting gonadotropin activity but also indirectly indicated a potential NPY stimulatory effect exerted on endogenous GnRH neurons in these animals. Because NPY is known to inhibit GnRH neurons excitability [26, 27], BIBP-mediated suppression of gonadotropin activity found in the present research has an opposite effect to the expected response for Y1R antagonist pretreatment. Moreover, our results also revealed that co-treatment with BIBP and orexin A further increased down-regulatory effects on LH release and $L H b$ mRNA level.

Nowadays, NPY receptor Y1 is known to be expressed on orexigenic neurons perikarya [28] Interestingly, in vitro studies revealed that Y1-specific antagonist eliminated orexin-A effects on GnRH secretion [11, 12]. NPY was reported in the literature to reduce orexin neuron activity [29]. Therefore, intraventricular BIBP infusion applied in immature females, as was done in our study, could result in increased endogenous orexinA secretion, which in turn diminished the level of LH secretion in these animals.

\section{Conclusions}

Our findings indicate that orexin-A exerts a down-regulatory effect on LH synthesis and release in immature female rats. Considering that Y1R-oriented down-regulation of endogenous NPY activity did not reverse the suppressive effect of exogenous orexin-A, it might be that NPY and orexin-A systems can operate independently to affect gonadotropin activity in the anterior pituitary gland of the immature female rat. Furthermore, an endogenous pro-gonadotropic NPY activity cannot also be excluded in these animals.

\section{Funding}

This research was supported by CMKP grant No. 501-1-1-27-29/07, 501-1-31-22-12, 501-1-31-22-16.

\section{Acknowledgments}

We would like to thank Dr. Ewa Wolińska-Witort for many years of friendly cooperation, help, and wise comments.

\section{References}

1. de Lecea L, Kilduff TS, Peyron C, et al. The hypocretins: hypothalamus-specific peptides with neuroexcitatory activity. Proc Natl Acad Sci U S A. 1998; 95(1): 322-327, doi: 10.1073/pnas.95.1.322, indexed in Pubmed: 9419374.

2. Sakurai T, Amemiya A, Ishii M, et al. Orexins and orexin receptors: a family of hypothalamic neuropeptides and $G$ protein-coupled receptors that regulate feeding behavior. Cell. 1998; 92(4): 573-585, doi: 10.1016/s0092-8674(00)80949-6, indexed in Pubmed: 9491897.

3. Chieffi S, Carotenuto M, Monda V, et al. Orexin System: The Key for a Healthy Life. Front Physiol. 2017; 8: 357, doi: 10.3389/fphys.2017.00357, indexed in Pubmed: 28620314.

4. Soya S, Sakurai T. Evolution of Orexin Neuropeptide System: Structure and Function. Front Neurosci. 2020; 14: 691, doi: 10.3389/fnins.2020.00691 indexed in Pubmed: 32754010.

5. Milbank E, López M. Orexins/Hypocretins: Key Regulators of Energy Homeostasis. Front Endocrinol (Lausanne). 2019; 10: 830, doi: 10.3389/fendo.2019.00830, indexed in Pubmed: 31920958.

6. Horvath TL, Diano S, van den Pol AN. Synaptic interaction between hypocretin (orexin) and neuropeptide $Y$ cells in the rodent and primate hypothalamus: a novel circuit implicated in metabolic and endocrine regulations. J Neurosci. 1999; 19(3): 1072-1087, indexed in Pubmed: 9920670.

7. López M, Seoane LM, García Md, et al. Neuropeptide Y, but not agouti-related peptide or melanin-concentrating hormone, is a target peptide for orexin-A feeding actions in the rat hypothalamus. Neuro- 
endocrinology. 2002; 75(1): 34-44, doi: 10.1159/000048219, indexed in Pubmed: 11810033.

8. Wang C, Han X, Guo F, et al. Orexin-A signaling in the paraventricular nucleus modulates spontaneous firing of glucose-sensitive neurons and promotes food intake via the NPY pathway in rats. Biochem Biophys Res Commun. 2018; 505(1): 162-167, doi: 10.1016/j.bbrc.2018.09.091, indexed in Pubmed: 30243725.

9. Jain MR, Horvath TL, Kalra PS, et al. Evidence that NPY Y1 receptors are involved in stimulation of feeding by orexins (hypocretins) in sated rats. Regul Pept. 2000; 87(1-3): 19-24, doi: 10.1016/s0167-0115(99)00102-0, indexed in Pubmed: 10710284

10. Jawiarczyk-Przybyłowska A, Bolanowski M. The role of orexin A in metabolic disturbances in patients with acromegaly. Endokrynol Pol. 2012; 63(6): 463-469, indexed in Pubmed: 23339004.

11. Iqbal J, Pompolo S, Sakurai T, et al. Evidence that orexin-containing neurones provide direct input to gonadotropin-releasing hormone neurones in the ovine hypothalamus. J Neuroendocrinol. 2001; 13(12): 1033-1041, doi: 10.1046/j.1365-2826.2001.00719.x, indexed in Pubmed: 11722699.

12. Russell SH, Small CJ, Kennedy AR, et al. Orexin A interactions in the hypothalamo-pituitary gonadal axis. Endocrinology. 2001; 142(12): 5294-5302, doi: 10.1210/endo.142.12.8558, indexed in Pubmed: 11713229.

13. Jöhren O, Brüggemann N, Dendorfer A, et al. Gonadal steroids differentially regulate the messenger ribonucleic acid expression of pituitary orexin type 1 receptors and adrenal orexin type 2 receptors. Endocrinology. 2003; 144(4): 1219-1225, doi: 10.1210/en.2002-0030, indexed in Pubmed: 12639903.

14. Kiyokawa M, Matsuzaki T, Iwasa T, et al. Neuropeptide $\mathrm{Y}$ mediates orexin A-mediated suppression of pulsatile gonadotropin-releasing hormone secretion in ovariectomized rats. J Med Invest. 2011; 58(1-2): 11-18, doi: 10.2152/jmi.58.11, indexed in Pubmed: 21372482.

15. Plant TM, Plant TM. Neurobiological bases underlying the control of the onset of puberty in the rhesus monkey: a representative higher primate. Front Neuroendocrinol. 2001; 22(2): 107-139, doi: 10.1006/frne.2001.0211, indexed in Pubmed: 11259134.

16. Paxinos G, Watson C. The Rat Brain in Stereotaxic Coordinates. Academic Press, New York 1986.

17. Untergasser A, Cutcutache I, Koressaar T, et al. Primer3 - new capabilities and interfaces. Nucleic Acids Res. 2012; 40(15): e115, doi: 10.1093/nar/gks596, indexed in Pubmed: 22730293.

18. Campbell RE, Grove KL, Smith MS. Gonadotropin-releasing hormone neurons coexpress orexin 1 receptor immunoreactivity and receive direct contacts by orexin fibers. Endocrinology. 2003; 144(4): 1542-1548, doi: 10.1210/en.2002-220958, indexed in Pubmed: 12639939

19. Gaskins GT, Moenter SM. Orexin a suppresses gonadotropin-releasing hormone (GnRH) neuron activity in the mouse. Endocrinol- ogy. 2012; 153(8): 3850-3860, doi: 10.1210/en.2012-1300, indexed in Pubmed: 22673226

20. Khazali H, Behzadfar M. Effect of Orexin Infusion into Third Ventricle on the GnRH and LH Secretions in the Prepubertal Rat. J Appl Sci. 2009; 9(10): 1936-1942, doi: 10.3923/jas.2009.1936.1942.

21. Small CJ, Goubillon ML, Murray JF, et al. Central orexin A has site-specific effects on luteinizing hormone release in female rats. Endocrinology. 2003; 144(7): 3225-3236, doi: 10.1210/en.2002-0041, indexed in Pubmed: 12810579 .

22. Martynska L, Wolinska-Witort E, Chmielowska M, et al. Effect of orexin A on the release of GnRH-stimulated gonadotrophins from cultured pituitary cells of immature and mature female rats. Neuropeptides. 2014; 48(4): 199-205, doi: 10.1016/j.npep.2014.05.005, indexed in Pubmed: 24931296.

23. Kohsaka A, Watanobe H, Kakizaki Y, et al. A significant participation of orexin-A, a potent orexigenic peptide, in the preovulatory luteinizing hormone and prolactin surges in the rat. Brain Research. 2001; 898(1): 166-170, doi: 10.1016/s0006-8993(01)02157-6.

24. Pu S, Jain MR, Kalra PS, et al. Orexins, a novel family of hypothalamic neuropeptides, modulate pituitary luteinizing hormone secretion in an ovarian steroid-dependent manner. Regul Pept. 1998; 78(1-3): 133-136, doi: 10.1016/s0167-0115(98)00128-1.

25. Döhler KD, Wuttke W. Changes with age in levels of serum gonadotropins, prolactin and gonadal steroids in prepubertal male and female rats. Endocrinology. 1975; 97(4): 898-907, doi: 10.1210/endo-97-4-898, indexed in Pubmed: 1193012

26. Roa J, Herbison AE. Direct regulation of GnRH neuron excitability by arcuate nucleus POMC and NPY neuron neuropeptides in female mice. Endocrinology. 2012; 153(11): 5587-5599, doi: 10.1210/en.2012-1470, indexed in Pubmed: 22948210.

27. Klenke U, Constantin S, Wray S. Neuropeptide Y directly inhibits neuronal activity in a subpopulation of gonadotropin-releasing hormone-1 neurons via Y1 receptors. Endocrinology. 2010; 151(6): 2736-2746, doi: 10.1210/en.2009-1198, indexed in Pubmed: 20351316.

28. Muroya S, Funahashi H, Yamanaka A, et al. Orexins (hypocretins) directly interact with neuropeptide Y, POMC and glucose-responsive neurons to regulate $\mathrm{Ca} 2+$ signaling in a reciprocal manner to leptin: orexigenic neuronal pathways in the mediobasal hypothalamus. Eur J Neurosci. 2004; 19(6): 1524-1534, doi: 10.1111/j.1460-9568.2004.03255.x , indexed in Pubmed: 15066149.

29. Fu LY, Acuna-Goycolea C, van den Pol AN. Neuropeptide Y inhibits hypocretin/orexin neurons by multiple presynaptic and postsynaptic mechanisms: tonic depression of the hypothalamic arousal system. J Neurosci. 2004; 24(40): 8741-8751, doi: 10.1523/JNEUROSCI.2268-04.2004, indexed in Pubmed: 15470140. 